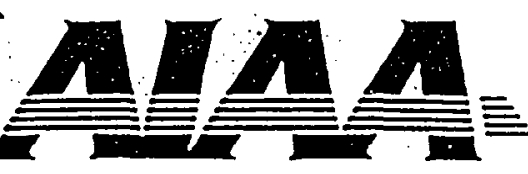

\title{
AIAA 99-3326
}

\section{A Front Tracking Scheme for High Density-Ratio Multi-Fluid Flows}

\author{
Erlendur Steinthorsson \\ Parker Hannifin Corporation \\ Gas Turbine Fuel Systems Division \\ Mentor, Ohio
}

Gretar Tryggvason

University of Michigan

Dept. of Mech. Eng. And Applied Mechanics

Ann Arbor, Michigan

\section{4th Computational Fluid Dynamics Conference$$
28 \text { June - } 1 \text { July, } 1999 \text { / Norfolk, VA }
$$ 


\section{A Front Tracking Scheme for High Density-Ratio Multi-Fluid Flows}

\author{
Erlendur Steinthorsson \\ Parker Hannifin Corporation \\ Gas Turbine Fuel Systems Divison \\ Mentor, Ohio
}

\author{
Gretar Tryggvason \\ University of Michigan \\ Dept. of Mech. Eng. and Applied Mechanics \\ Ann Arbor, Michigan
}

\begin{abstract}
Numerical simulations of high-density ratio, incompressible, multi-fluid flows are presented. The simulations are done using the front tracking scheme of Univerdi and Tryggvason (," J. of Comp. Phys., Vol. 100, pp. 25-). To facilitate simulations of density ratio flows, the governing equations are discretized using second-order accurate upwind differencing. Also, a modification to the time stepping scheme used in earlier work is introduced that also improve the capability of the scheme to handle high density-ratio flows. Results are presented for density ratios as high as 10,000 to one.
\end{abstract}

\section{Introduction}

In recent years, significant advances have been made in numerical simulations of multi-fluid and multi-phase flows. Recent applications of general interest include collisions of droplets, breakup of droplets, droplets or non-deformable objects falling into liquid under gravity, a multitude of bubbles rising through liquid under gravity, and flow of a multitude of nondeformable particles in liquids.

One category of multi-fluid flows of interest is the atomization of liquids. Atomization is the breakup of bulk liquid into a vast number of small droplets. It requires energy input as the total amount of surface energy in a spray of droplets is significantly higher than that of the bulk liquid. The required energy is imparted to the liquid, for example, by accelerating it through a small orifice to form a jet that subsequently breaks up due to interaction between the liquid and air; or by blasting a liquid sheet with high-speed air. The fluid mechanics of the break-up of jets and liquid sheets are complicated, not thoroughly understood. The breakup is extremely hard to study experimentally and not yet predictable by any numerical method. The ultimate goal of the current research is to develop the capability to study such break-up by using numerical simulations.

Copyright (C) by the American institute of Aeronautics and Astronautics
Several numerical methods have been developed to simulate multi-fluid flows. The earliest ones are the classical Marker-in-Cell (MAC) method, where marker particles, identifying each fluid, are advected with the flow, and the Volume-of-Fluid method (VOF) where a marker function (the volume fraction) is advected with the flow. These methods are still popular and improvements are still being developed (see e.g., Brackbil, et al., 1992). The VOF method is available for use in engineering flow simulations in commercial codes such as Fluent (Fluent Inc., 1998) and CFX (AEA Technology, 1998). Various applications of the VOF method have been reported in the literature. A method that is related to the VOF method is the recently introduced level-set method (Sussman, et al., 1994). Another approach is to use Lagrangian methods, such as the Arbitrary Lagrangian Eulerian (ALE) method (Hirt, et al., 1970), where a grid system follows and deforms with the fluids. This method has the advantage that the interface between two fluids is always sharp and well defined. One characteristic of the method is that only a limited amount of deformation of the computational grid can be tolerated before re-gridding is needed. In addition, automatic handling of changing interface topology is difficult. Nonetheless, this approach is attractive for certain types of problems. An impressive example of the use of ALE method that was reported recently is the simulation of a multitude of arbitrary shaped, non-deformable particles flowing suspended in a liquid (Hu, 1998). One additional class of methods are "interface fitting" methods, where a grid is modified to align with the interface while a stationary grid is otherwise used. Glimm and co-workers (Glimm, 1991) have investigated these types of methods.

In this paper, we focus on a method that can be considered a hybrid between the interface capturing methods and the interface fitting method. This method has been called a "Front Tracking method." It was developed primarily by 
Tryggvason and co-workers (Univerdi and Tryggvason, 1992, Nobari and Tryggvason, 1994). This method uses a single fluid formulation, similar to the VOF method but uses a chain or a network of marker particles to mark the location of the interface between two fluids. This method has primarily been used in highly resolved simulations of flows involving bubbles and droplets at moderate density ratios. It has proved capable of producing accurate results on relatively coarse grids. The method has also been applied to other types of interface problems such as dendritic solidification and the suction of a single biological cell into a microscopic pipette. Here we will demonstrate the application of a modified version of the method to high density-ratio flows such as encountered in the atomization of liquids. The test case we consider is the interaction between a liquid sheet and surrounding air. Results for density ratios of 1000 and 10000 are presented.

Simulations of high density-ratio multi-fluid flows on fixed grid systems are difficult primarily for the reason that the interface between two fluids represent a discontinuity in density, viscosity and other fluid properties and that this discontinuity must be captured on a grid system with finite resolution. More succinctly, the discrete grid system can only support a finite number of frequencies or Fourier modes whereas an infinite number of modes are required to represent the discontinuity. When the discrete solution is advanced in time, the discontinuity gives rise to high frequency modes that get "aliased" to low frequency modes that are supported by the grid system. Gradually, this gives rise to errors that contaminate the computed solution. With sufficient meshresolution the physical viscosity damps all unphysical high frequency errors before they get aliased to the lower frequencies. For less resolved simulations, we must address the difficulty more directly. First, we can attempt to use some type of a discretization that is capable of "capturing" the discontinuity without producing high frequency errors. Second, we can attempt to spread the discontinuity over a handful of cells rather than attempt to capture it as a discontinuity. In this paper, we focus on the first approach. In particular, we test the abilities of an upwind scheme that we proposed in earlier work in simulations of high-density ratio flows. We introduce a modification to our earlier method that significantly enhances its capability to simulate high-density ratio flows.

In the remainder of this paper we first review the governing equations used to describe the multifluid flow and briefly explain the ideas behind the front tracking scheme of Tryggvason and coworkers. Thereafter, the discretization of the governing equations and the current solution algorithm are reviewed. Finally, the results from tests are presented and discussed.

\section{Governing Equations and the Front Tracking Method}

The flow of two immisicible fluids is taken to be incompressible. Here only axisymmetric flows are considered. The governing equations are cast a single set of equations (one fluid formulation) using a variable density and viscosity as follows:

$$
\begin{gathered}
\frac{\partial \rho u}{\partial t}+\frac{1}{r} \frac{\partial}{\partial r}\left(\rho r u^{2}\right)+\frac{\partial}{\partial z}(\rho u v)-\frac{\rho w^{2}}{r}= \\
-\frac{\partial p}{\partial r}+\frac{1}{r} \frac{\partial}{\partial r}\left(2 \mu r \frac{\partial u}{\partial r}\right) \\
+\frac{\partial}{\partial z}\left(\mu\left(\frac{\partial u}{\partial z}+\frac{\partial v}{\partial r}\right)\right)+f_{r} \\
\frac{\partial \rho v}{\partial t}+\frac{1}{r} \frac{\partial}{\partial r}(\rho r u v)+\frac{\partial}{\partial z}\left(\rho v^{2}\right)=-\frac{\partial p}{\partial z} \\
+\frac{\partial}{\partial z}\left(2 \mu \frac{\partial v}{\partial z}\right)+\frac{1}{r} \frac{\partial}{\partial z}\left(\mu\left(\frac{\partial u}{\partial z}+\frac{\partial v}{\partial r}\right)\right)+f_{z} \\
\frac{\partial \rho w}{\partial t}+\frac{1}{r} \frac{\partial}{\partial r}(\rho r u w)+\frac{\partial}{\partial z}(\rho v w)= \\
\frac{1}{r^{2}} \frac{\partial}{\partial r}\left(\mu r^{3} \frac{\partial}{\partial r}\left(\frac{w}{r}\right)\right)+\frac{\partial}{\partial z}\left(\mu \frac{\partial w}{\partial z}\right)
\end{gathered}
$$

The equations are complemented by the incompressibility condition

$$
\frac{1}{r} \frac{\partial r u}{\partial r}+\frac{\partial v}{\partial z}=0
$$


The forcing term

$$
\vec{f}=\left(f_{r}, f_{z}\right),
$$

arises due to surface tension at the interface between two fluids or two phases. It can be written as an integral over a Dirac delta-function (Nobari and Tryggvason, 1994b), i.e.,

$$
\vec{f}=\int_{F} \frac{1}{r}\left(\frac{\partial}{\partial s}(r \sigma \vec{t})-\sigma \vec{i}_{r}\right) \delta_{r z}\left(\vec{r}-\vec{r}_{f}\right) d s
$$

where $\sigma$ is the surface tension, $\vec{t}$ is the unit tangent to the front and $\vec{i}_{r}$ is a unit vector in the radial direction. $\delta_{r z}$ is the two dimensional delta-function, $\vec{r}=(r, z)$ and $\vec{r}_{f}$ is a point on the front. The integration is over the interface between the two fluids. Note, due to the assumption of axisymmetry, no surface tension force acts in the azimuthal direction. For further details on the one-fluid formulation, see Ref. 17.

The density, $\rho$, and viscosity, $\mu$, are taken to be constants for each fluid. Thus, the evolution of the density and viscosity fields can be described by

where

$$
\frac{D \rho}{D t}=0 \text { and } \frac{D \mu}{D t}=0
$$

$$
\frac{D \varphi}{D t}=\frac{\partial \varphi}{\partial t}+\vec{u} \cdot \nabla \varphi
$$

represents the total or "material" derivative. The two equations simply state that the density and viscosity of a fluid particle is constant.

Instead of attempting to solve Eq. (3) everywhere, as in the VOF method and the level-set method, it is sufficient to track the interface between the two fluids or phases across which the properties change. The interface moves with the local fluid velocity, i.e.,

$$
\frac{d \vec{r}_{f}}{d t}=\vec{u}
$$

where $r_{f}$ is a point on the interface and $\vec{u}$ is the local fluid velocity. This is the approach taken in the Front Tracking Method of Univerdi and Tryggvason (Ref. 17). In essence, this approach is equivalent to tracing the characteristics in order to update the density and viscosity fields. Here it is only noted that one of the strengths of the front-tracking scheme of Unverdi and Tryggvason is that it automatically captures interactions between neighboring (nonintersecting) fronts without any special treatment. For more details on the mathematical formulation of the one-fluid approach and the Front Tracking Method, see Ref. 17.

\section{Discretization of Governing Equations}

The governing equations, Eq. (1), (2) and (4), are discretized in space using a staggered mesh and integrated in time using a four-stage RungeKutta-based projection method. An interface between two fluids is represented by a linked list of points (an unstructured triangular mesh in three-dimensions) that is called the front (see Fig. 1). The front is continuously adapted to ensure appropriate resolution at all times. Adaptation involves adding and deleting nodes from the front when the distance between nodes becomes too large or too small, relative to the grid spacing.

Spatial Discretization: Two types of discretization schemes have been used with the front tracking scheme for the convective terms of the governing equations. The first is a central differencing scheme that has been used successfully in simulations of various flows of bubbles and droplets. The second is a secondorder accurate, Godunov type upwind scheme. The upwind scheme is based on a Godunov type scheme suggested by Bell et al. (1989), known as the BCG scheme. The upwind scheme was adapted for use in staggered meshes and multi-stage time-stepping schemes by Steinthorsson et al. (1997) and was found to perform well on relatively coarse grids. In this work, which focuses on high density-ratio flows, we consider only the upwind scheme.

To describe the upwind schemes we consider a conservation cell for the $r$-momentum and write out the fluxes through the left and bottom boundaries of the cell. In the staggered mesh the r-momentum cell resides at, say, $(i+1 / 2, j)$, with the left and bottom faces of the cell centered at $(i, j)$ and $(i+1 / 2, j-1 / 2)$, respectively. Fluxes through other conservation cells are formulated in an equivalent manner. Recalling that in the staggered mesh, $\rho$ resides at $(i, j), u$ resides at $(i+1 / 2, i)$ and $v$ resides at $(i, j+1 / 2)$, the 
upwind-weighted r-momentum flux through the cell face at $(i, j)$ is computed as

$$
F_{i, j}^{u}=\rho_{i, j}\left(u_{i, j}\right)^{2}
$$

where

$$
u_{i j}= \begin{cases}u^{L} & \text { if } u^{L} \geq 0, u^{L}+u^{R} \geq 0 \\ 0 & \text { if } u^{L}<0, u^{R}>0 \\ u^{R} & \text { otherwise }\end{cases}
$$

The "left" and "right" states, $u^{L}$ and $u^{R}$ are extrapolated from the cells on either side of the cell face using "central-differenced limited slopes" (see Bell, et al., 1989) for an overall second-order accurate scheme. The rmomentum flux through the cell face at $(i+1 / 2, j-$ $1 / 2$ ) is computed as

$$
F_{i+\frac{1}{2}, j-\frac{1}{2}}^{u}=\frac{1}{2}\left(v_{i, j-\frac{1}{2}}+v_{i+1, j-\frac{1}{2}}\right) \rho_{i+\frac{1}{2}, j-\frac{1}{2}} u_{i+\frac{1}{2}, j-\frac{1}{2}}
$$

where $\rho_{i+\frac{1}{2}, j-\frac{1}{2}}$ and $u_{i+\frac{1}{2}, j-\frac{1}{2}}$ are computed by first extrapolating from "left" and "right" to the cell boundary and then choosing the right or left state based on the sign of $\left(v_{i, j-\frac{1}{2}}+v_{i+1, j-\frac{1}{2}}\right)$.

The scheme, as written, is not a pure upwind scheme since the density that is used in the computation of the r-momentum flux through the constant-r face of the conservation cell resides at the cell face. In the current work, this density is the average density in a cell centered at the boundary face. This can negatively impact the stability characteristics of the scheme. A better approach is to extrapolate the density from the upwind side. This has not yet been implemented in our computer codes. However, the front tracking scheme allows an easy way to accomplish this as point and average values of density can be computed for any point or region in the domain. This will be addressed in future work.

Time Stepping Scheme: The governing equations are integrated in time using a four stage Runge-Kutta scheme. Both the momentum equations and the front are advanced using the same scheme and the incompressibility constraint is enforced in every stage using a projection method. Before writing out the multi-stage scheme, we consider an Euler-explicit discretization using a one- dimensional momentum equation to illustrate the details of a single stage.

Using Euler-explicit time stepping, the discretized one-dimensional momentum equation can be written as

$$
(\rho u)_{i+\frac{1}{2}}^{n+1}=\frac{1}{2}\left(\rho_{i}^{n}+\rho_{i+1}^{n}\right) u_{i+\frac{1}{2}}^{n}-\frac{\Delta t}{\Delta x_{i+\frac{1}{2}}}\left(F_{i+1}^{u}-F_{i}^{u}\right)
$$

where $F_{i}{ }_{i}$ is the flux through the cell face at (i). The velocity at the new time step (or new stage in a Runge-Kutta scheme) is then computed as

$$
u_{i+\frac{1}{2}}^{n+1}=\frac{(\rho u)_{i+\frac{1}{2}}^{n+1}}{\rho_{i+\frac{1}{2}}^{n+1}}
$$

Here, the density at the new time level can be obtained from the values in the "density cells" that are computed based on the location of the front at the new time level, i.e.

$$
\rho_{i+\frac{1}{2}}^{n+1}=\frac{1}{2}\left(\rho_{i}^{n+1}+\rho_{i+1}^{n+1}\right)
$$

This approach has been used with the front tracking scheme in earlier papers. It has been found to work well for low to moderate density ratios. However, with this approach, there is a disconnection between the density field and the velocity field. As a result, for instance, a uniform velocity field and a discontinuous density field at time level $n$ will produce a non-uniform velocity field at time level $n+1$. The perturbation in the velocity field is proportional to the jump in density and the time step size. For high densityratios, the time step required to keep the perturbation sufficiently small becomes unacceptable for all but the smallest simulations.

To overcome this disconnection between the density and velocity fields, we introduce a temporary density field that is used only for updating the velocity at the new time level. The temporary density field is computed by utilizing the continuity equation and discretizing it in the same manner as the momentum equation. Thus, we compute the new density in the r-momentum cell (centered at $i+1 / 2$ ) as

$$
\rho_{i+\frac{1}{2}}^{n+1}=\frac{1}{2}\left(\rho_{i}^{n}+\rho_{i+1}^{n}\right)-\frac{\Delta t}{\Delta x_{i+\frac{1}{2}}}\left(\rho_{i+1}^{n} u_{i+1}-\rho_{i}^{n} u_{i}\right)
$$

where $u_{i}$ is evaluated the same way as for the momentum equation. Note that the temporary density field is "co-located" with the velocity field 
and that, on a staggered mesh in two dimensions, two such fields are computed; one for each velocity component. Using this approach, a uniform velocity field at the new time level is ensured, regardless of the density field (in the absence of surface tension). The restriction on the time step that was produced by the earlier approach is removed. In a sense, the overall scheme is now closer to schemes that advect a volume fraction or a color function. However, the density field will remain sharp at all times (as sharp as desired) and the front is still used to evaluate source terms due to surface tension.

The time stepping scheme can be written as follows:

$$
\begin{aligned}
& U_{0}=U^{n}, \quad X_{0}=X^{n} \\
& X_{k}=X_{0}+\alpha_{k} \Delta t R_{x}\left(X_{k-1}, U_{k-1}\right) \text {. } \\
& U_{k}=\mathbf{P}\left(U_{0}+\alpha_{k} \Delta t R_{u}\left(X_{k-1}, U_{k-1}\right)\right) \\
& \text { for } k=1, \ldots, 4 ; \quad \alpha=\left(\begin{array}{llll}
\frac{1}{2} & \frac{1}{2} & 1 & 1
\end{array}\right) \\
& X^{n+1}=X_{0}+\Delta t \sum_{k=1}^{4} \beta_{k} R_{x}\left(X_{k-1}, U_{k-1}\right) \\
& U^{n+1}=\mathbf{P}\left(U_{0}+\Delta t \sum_{k=1}^{4} \beta_{k} R_{u}\left(X_{k-1}, U_{k-1}\right)\right) \\
& \beta=\left(\begin{array}{llll}
\frac{1}{6} & \frac{1}{3} & \frac{1}{3} & \frac{1}{6}
\end{array}\right)
\end{aligned}
$$

where $X$ and $U$ represent the solution vectors for the nodes on the front and the velocity field, respectively, and $R_{X}$ and $R_{U}$ represent the residuals of the respective governing equations. The superscript " $n$ " denotes the time level. The operator $\mathbf{P}$ is the projection operator that is used to ensure that the velocity field is divergence free (see Ref. 1 for a review of the ideas behind projection methods). Note that in every stage of the time stepping scheme the velocities of the nodes of the front need to be evaluated. This is done using linear interpolation in the cell in which the node resides.

Reconstruction of density field: After the front has been advanced in time, it is necessary to reconstruct the density field. For the vast majority of cells, this is trivial since most are located far from the interface where the density does not change. For cells in the immediate neighborhood of the front, the density field is reconstructed in a special manner. In essence, the jumps in density and viscosity, as well as the forces due to surface tension, are distributed in a conservative manner to cells within a narrow interval around the front. This produces an interface that has a finite thickness. The thickness is proportional to the grid spacing and is constant in time. The reconstruction of the density field is accomplished by constructing a special indicator function on cells surrounding the front. Once the indicator function has been constructed, it is used to determine the density and other fluid properties in the neighborhood of the front. Due to the method used to construct the indicator function, interactions between neighboring (non-intersecting) fronts are handled automatically. No special treatment is needed.

\section{Description of Test Problem}

A special prototypical flow is used in this study that has relevance to atomization. The flow consists of an infinite annular sheet of highdensity fluid and of finite thickness surrounded by a high-speed low-density fluid (see Fig. 2). For convenience, we refer to the high-density fluid as "liquid" and the low-density fluid as "air". For most of the test cases, the initial flow field is perturbed by introducing a body force that varies sinusoidally in the axial direction. After a short time, the body force is turned off and the flow field is allowed to evolve unforced. In one case, a periodic disturbance in the air is introduced at time zero. The disturbance in the air produces a disturbance in the liquid sheet.

The problem being simulated can be described in terms of the non-dimensional parameters defined by Liao, et al $(1998$ a,b). In their work, the non-dimensional parameters that are relevant for the current test problem are defined as

$$
\begin{aligned}
& W e_{i}=\frac{\rho_{i} U_{i}^{2} R_{b}}{\sigma}, \\
& W e_{o}=\frac{\rho_{o} U_{o}^{2} R_{b}}{\sigma}, \\
& W e_{l}=\frac{\rho_{l} U_{l}^{2} R_{b}}{\sigma},
\end{aligned}
$$




$$
\begin{gathered}
g_{i}=\frac{\rho_{i}}{\rho_{l}}, \quad g_{o}=\frac{\rho_{o}}{\rho_{l}}, \quad h=\frac{R_{a}}{R_{b}}, \\
\bar{k}=k R_{b} \quad \varpi=\frac{\omega R_{b}}{U_{1}} \\
\text { density ratio }=1 / g
\end{gathered}
$$

where $U_{i}$ and $U_{o}$ are the axial velocities of inner and outer air, respectively, $U_{l}$ is the velocity of the liquid, $\rho_{i}$ and $\rho_{o}$ are the densities of the inner and outer air, respectively, $R_{a}$ and $R_{b}$ are the inner and outer radii of the annular liquid sheet. The initial disturbance of the interface is characterized by a non-dimensional wave number, $k$, and by an amplitude, $a$. In the present work, we also have Reynolds number, defined in terms of $R_{b}$, air properties and velocity; and viscosity ratios, as only viscous flow is considered. For all the cases considered, the inner and outer air velocities are equal.

\section{Results}

Results from two simulations of the prototype flow described above are presented. In one, a density ratio of 1000 is used and surface tension is assumed to be zero, corresponding to infinite Weber number. In the second case, the density ratio is taken to be 10000 and the Weber number, based on the air density and air velocity, is 2.0 . In both cases, the velocity of the liquid is $U_{L}=0.1414 U_{\text {air, }}$ where $U_{a i r}$ is the initial velocity of the air that is also used as a reference velocity in the non-dimensionalization of the equations. In both cases, the liquid sheet is perturbed using a sinusoidal body force that acts for a short time. Both simulations are performed on a $256 \times 256$ grid system.

In the first case, the aerodynamic forces that act on the liquid sheet are destabilizing since the restorative effects of the surface tension forces are absent. Thus, we expect the aerodynamic forces to drive the deformation of the sheet after an initial transient where the initial disturbance of the sheet dominates. This is seen in Figure 2 , which shows the deformation of the liquid sheet at several instances in time. The amplitude of the deformation continually increases as expected. Initially, the deformation is nearly sinusoidal. Quickly, however, a "cusp" starts developing in the outer interface, where highspeed air impinges on the sheet. As the amplitude of the deformation grows, the shape of the sheet becomes more complicated. The sheet thins as it deforms and it becomes more easily distorted by the low-density but high velocity air.

For the second case, the density of the liquid is higher than in the first case and surface tension acts at the interface. Based on the Weber number, the effects of aerodynamic forces are comparable to the surface tension effects. However, the inertia of the liquid sheet is an order of magnitude larger than in the first case. Thus, we expect any initial disturbance of the velocity of the liquid phase to completely dominate the deformation of the sheet.

Figure 3 shows the deformation of the sheet. As the figure shows, the shape of the sheet remains more or less sinusoidal to the end of the simulation. The surface tension eliminates any formation of a "cusp" in the interface that was seen in the earlier case.

As a final figure, we show a close-up view of the velocity field near the liquid sheet in case shown in Fig. 2d. This is shown in Figure 4. In the figure, the relative velocity between the sheet and the air is shown. The figure shows the air recirculating in the troughs formed by the distorted sheet. It illustrates the capture of high velocity gradients that naturally occur near the high-density sheet. The figure also shows the air impinging on the sheet and causing local deformation of the interface as well as the formation of boundary layers on the air-side of the liquid-air interfaces.

\section{Summary and Conclusions}

In this paper, we have demonstrated the use of the Front Tracking scheme of Univerdi and Tryggvason in simulations of high density-ratio multi-fluid flows. In this work, we used an upwind scheme to discretize the advective terms of the governing equations, coupled with a four-stage Runge-Kutta scheme to advance the computed solution in time. Compared to earlier applications of the Front Tracking scheme, a modified approach is used to advance the velocity field from one time level to the next. In earlier applications, the velocity field was computed as follows: First, the density field at the new time level was computed by advancing the front in time. Then a momentum field at the new time level was computed from the velocity field and density field at the old time level, using the discretized momentum equations. Finally, the new velocity field was computed from the 
new momentum field and the new density field, averaging as needed in the staggered mesh. In the current approach, the density field at the new time level is computed by advancing the front in time as before. Likewise, the momentum field is computed as before. However, a temporary density field, that is co-located with the velocity field, is computed by advancing the continuity equation in time and using the same differencing scheme as in the momentum equations. This density field is used solely to compute the velocity field at the new time level. With this modification, the overall scheme is less sensitive to high-density ratios than the original scheme. Simulations of flows with density ratios of 1000 and 10000 have been computed. Further improvements in the differencing scheme are possible and have been identified. These will be pursued in future work.

\section{References}

1. Bell, J.B., Colella, P., and Glaz, H.M., 1989, "A Second Order Projection Method for the Incompressible Navier Stokes Equations," J. of Computational Physics, vol. 85, pp. 257-283.

2. Brackbill, J,U,, Kothe, D.B., and Zemach, C., 1992, "A Continuum Method for Modeling Surface Tension," J. of Computational Physics, vol. 100, pp. 335-354.

3. Crapper, G.D., Dombrowski, N., and Pyott, G.A.D., 1975, "Kelvin-Helmholtz Wave Growth on Cylindrical Sheets," J. of Fluid Mech., V. 68, pp. 497-502.

4. E, W., and Liu, J.-G., 1996, "Vorticity Boundary Condition and Related Issues for Finite Difference Schemes," J. of Computational Physics, vol. 124, pp. 368-382.

5. Glimm, J., McBryan, O. A., Menikoff, R., and Sharp, D.H., 1987, SIAM J. Sci. Stat. Comput., Vol. 7, p. 230.

6. Glimm, J., Grove, J., Lindquist, B., McBryan, O. A., and Tryggvason, G.; 1988, "Bifurcations of Tracked Scalar Waves," SIAM J. Sci. Stat. Comput., Vol. 9, p. 61

7. Glimm, J., 1991, "Nonlinear and Stochastic Phenomena: The Grand Challenge for Partial Differential Equations," SIAM Review, Vol. 33, pp. $625-643$.

8. Hirt, C.W., Cook, J.L. and Butler, T.D., 1970,

"A Lagrangian Method for Calculating the
Dynamics of an Incompressible Fluid with Free Surface," J. of Comp. Phys., Vol. 5, pp. 103-124.

9. Hu, H.H., 1998, "Simulation of Particulate Flows of Newtonian and Viscoelastic Fluids," Proc., Third Intern. Conf. On Multiphase Flow, ICMF'98, Lion, France.

10. Liao, Y., Sakman, A.T., Jeng, S.M., Jog, M.A., and Benjamin, M., 1998, A comprehensive Model to Predict Simplex Atomizer Performance, To be presented at ASME/IGTI Turbo Expo, 1998, Stockholm, Sweden, June 5-8, 1998.

11. Liao, Y., Jeng, S.M., Jog, M.A., and Benjamin, M., 1998, Disintegration of an Annular Liquid Sheet Subject to Swirling Air Streams, to be presented at ILASS Americas, $11^{\text {th }}$ Conference on Liquid Atomization and Spray Systems, Sacramento, California, May 1998.

12. Nobari, M.R.H, and Tryggvason, G.; "Numerical Simulations of Drop Collisions," ICOMP Report 94-23. Also, NASA TM-106751 and AIAA-94-0835.

13. Nobari, M.R.H, and Tryggvason, G.; "The Flow Induced by the Coalescence of Two Initially Stationary Drops," ICOMP Report 94-24, October 1994. Also, NASA TM-106752.

14. Peskin, C.S.,"Numerical Analysis of Blood Flow in the Heart," J. of Comp. Phys., Vol, 25, p. 220. 1977.

15. Steinthorsson, E., Ajmani, K., Tryggvason, G. and Benjamin M. (1997) "Numerical Simulations of Multi-Fluid Flows in Fuel Atomizers," ASME paper FEDSM97-3549.

16. Sussman, M., Smereka, P. and Osher, 1994, "A Level Set Approach for Computing Solutions to Incompressible Two-Phase Flows," J. of Comp. Phys., Vol. 114, pp. 146-159.

17. Univerdi, S. O. and Tryggvason, G. (1992), "A Front-Tracking Method for Viscous, Incompressible Multi-Fluid Flows," J. of Comp. Phys., Vol. 100, pp. 25-37.

18. Zaleski, S., Jie, L., 1997, Direct Simulations of Spray Formation, proceedings, ICLASS-97, August 18-22, Seoul, South Korea. 

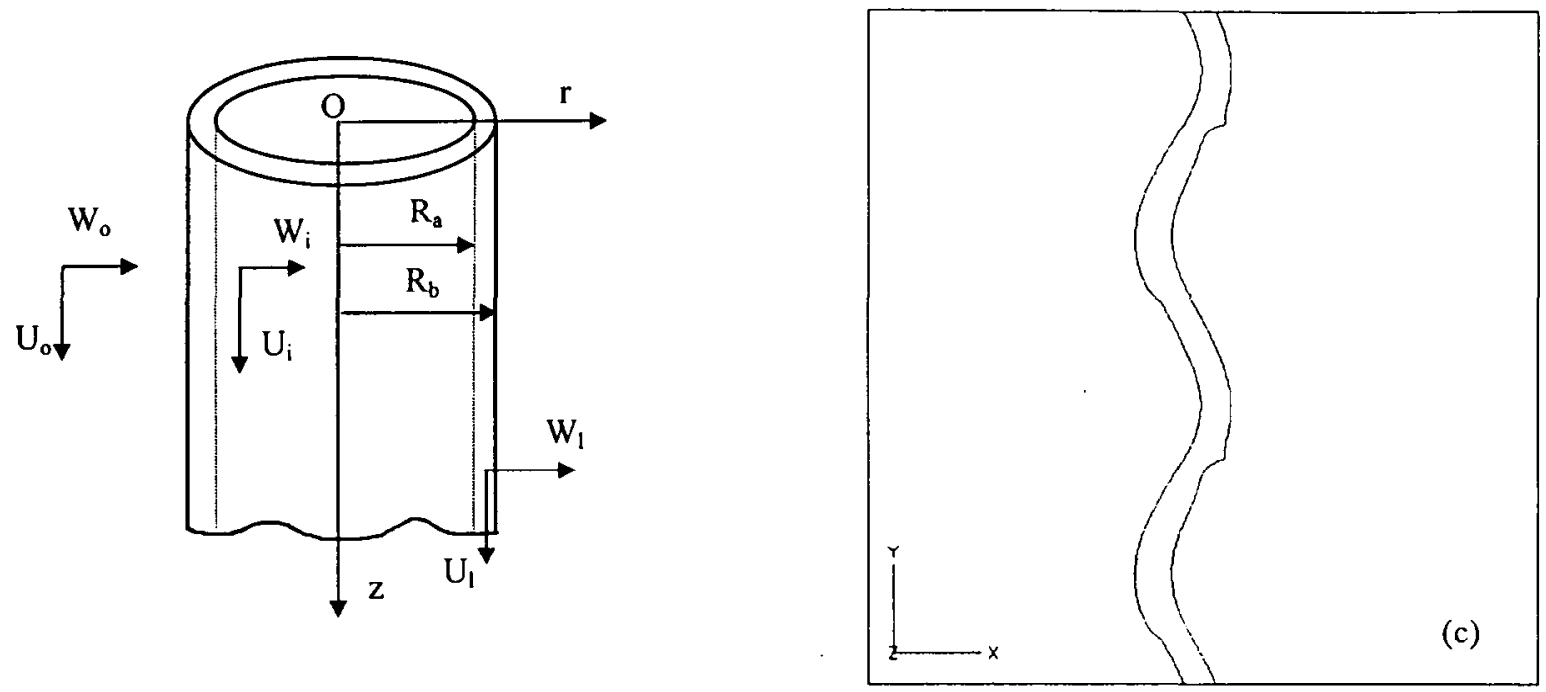

Figure 1: Schematic of an annular liquid sheet.
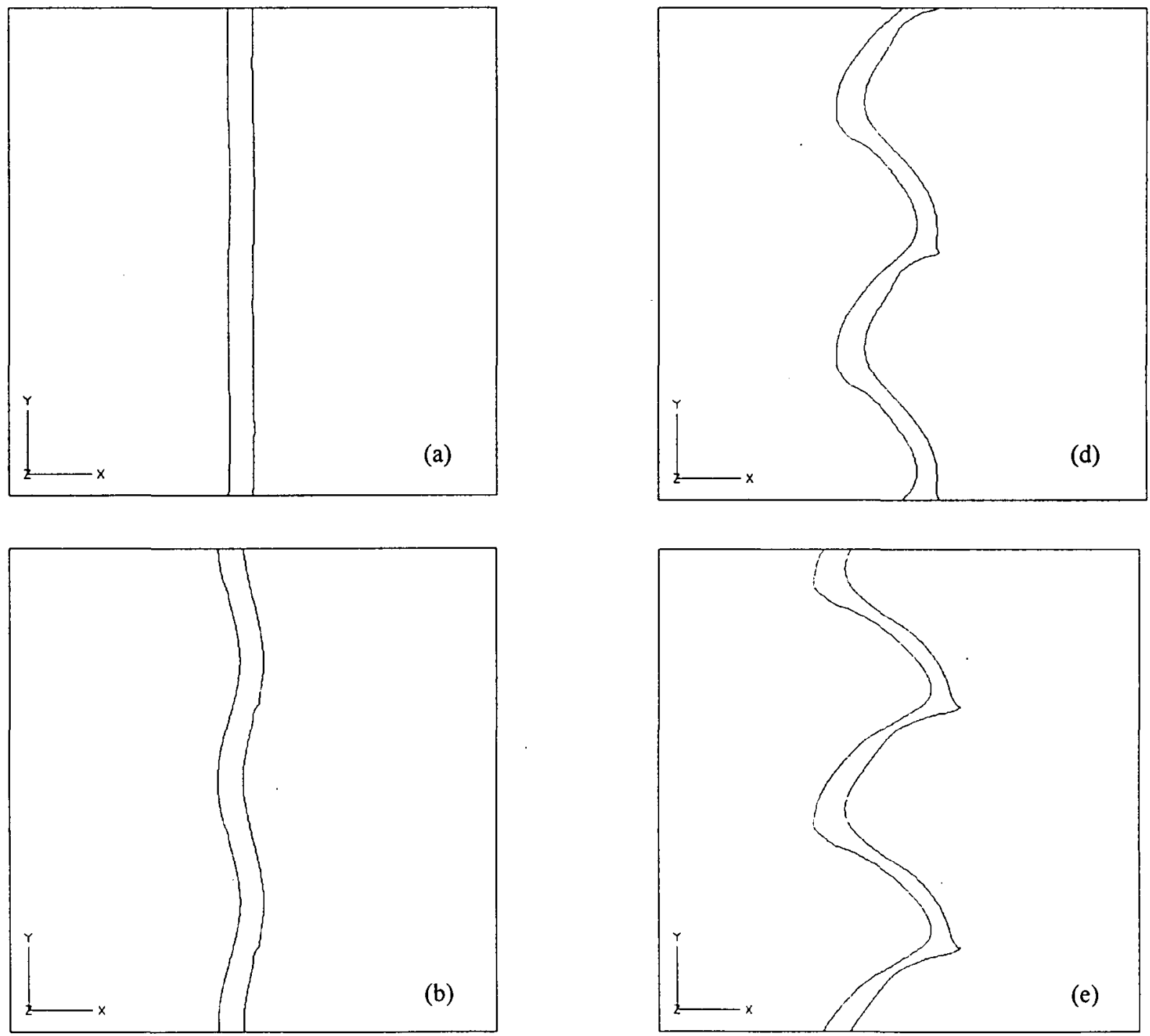

Figure 2. Deformation of an annular liquid sheet (axisymmetric); $\rho_{\mathrm{L}} / \rho_{\text {air }}=1000, \mu_{\mathrm{L}} / \mu_{\mathrm{air}}=1000$, $U_{\mathrm{L}} / U_{\text {air }}=0.1414, \operatorname{Re}=8333$, no surface tension $(1 / \mathrm{We}=0)$ : (a) $\mathrm{t}=0.01$, (b) $\mathrm{t}=1.0$, (c) $\mathrm{t}=2$, (d) $\mathrm{t}=3$, (e) $\mathrm{t}=4$. 
(c)1999 American Institute of Aeronautics \& Astronautics

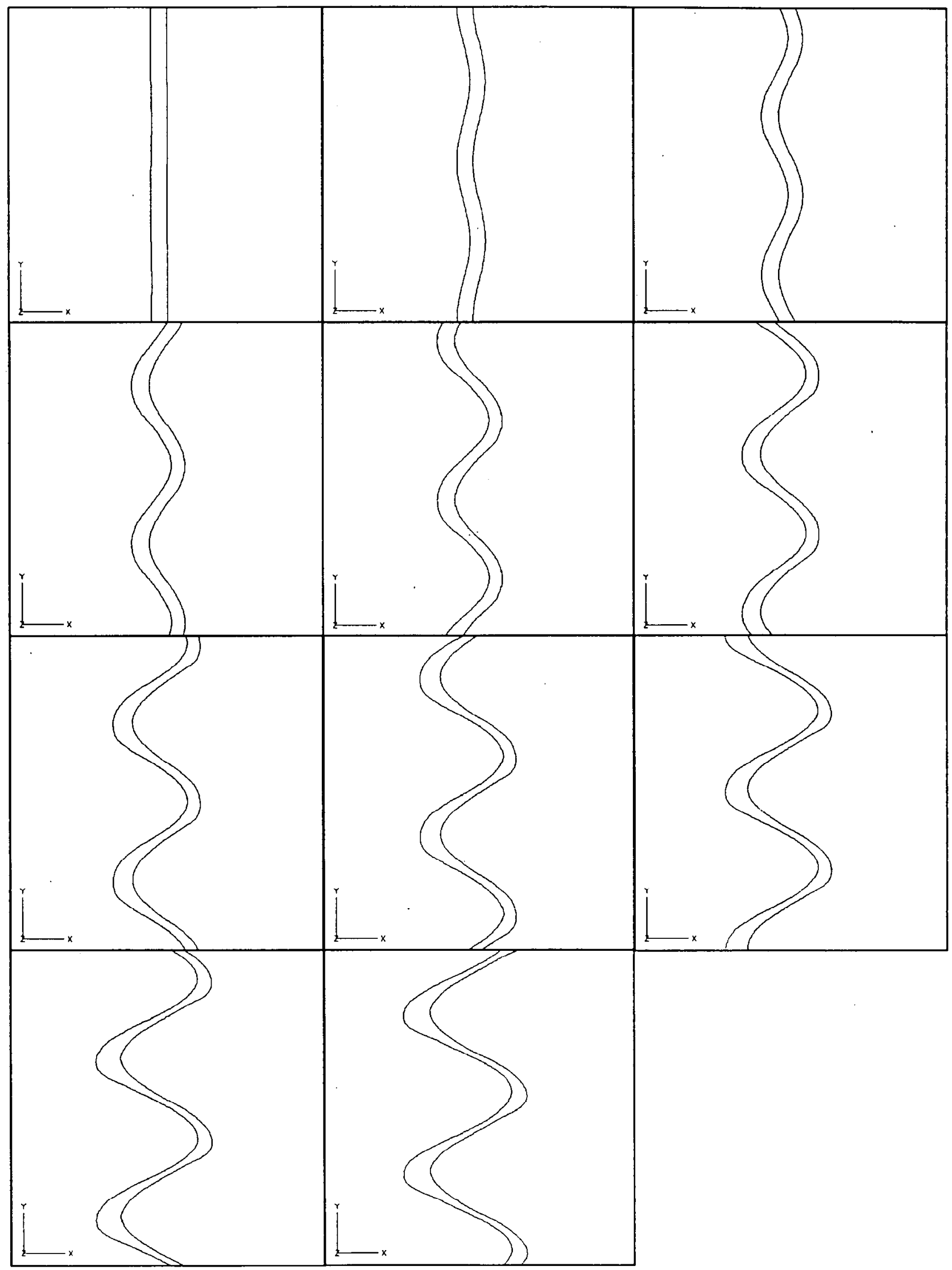

Figure 3. Deformation of an annular liquid sheet (axisymmetric); $\rho_{\mathrm{L}} / \rho_{\mathrm{air}}=10,000, \mu_{\mathrm{L}} / \mu_{\mathrm{air}}=1000$, $\mathrm{U}_{\mathrm{L}} / \mathrm{U}_{\text {air }}=0.1414, \mathrm{Re}=8333$, We $=2$, at non-dimensional times of $0.1,1,2,3,4,5,6,7,8,9$ and 10 , 


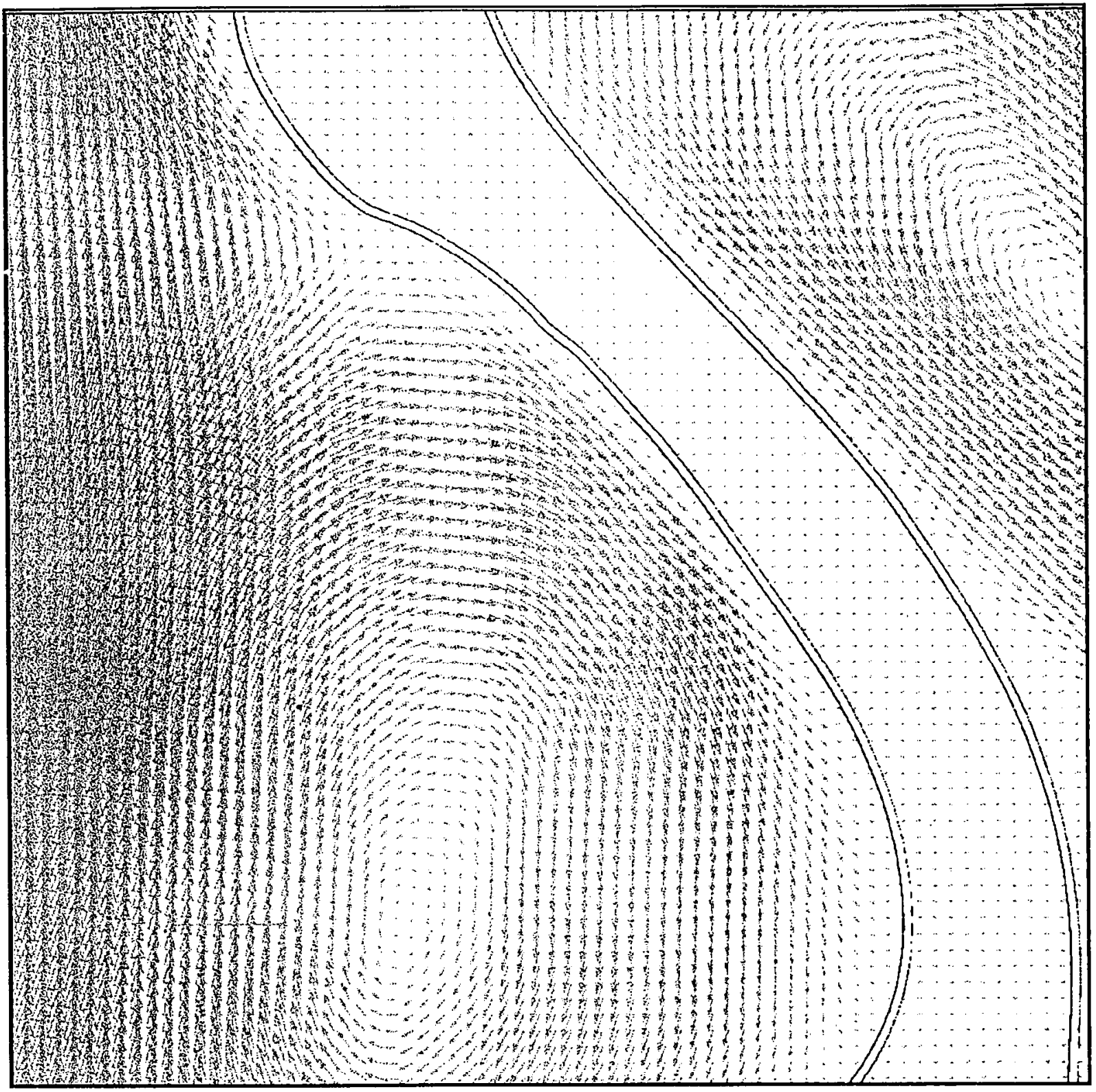

Figure 4. Vectors of relative velocity near liquid sheet 